\title{
Study of a Two-Step Centrifugation Protocol for Concentrating Cells and Growth Factors in Bovine Platelet-Rich Plasma
}

\author{
Claudia M. Gutiérrez, Catalina López, Carlos E. Giraldo, and Jorge U. Carmona \\ Grupo de Investigación Terapia Regenerativa, Departamento de Salud Animal, Universidad de Caldas, Manizales, Colombia \\ Correspondence should be addressed to Jorge U. Carmona; carmona@ucaldas.edu.co
}

Received 2 July 2017; Accepted 1 October 2017; Published 30 October 2017

Academic Editor: Pedro J. Ginel

Copyright (C) 2017 Claudia M. Gutiérrez et al. This is an open access article distributed under the Creative Commons Attribution License, which permits unrestricted use, distribution, and reproduction in any medium, provided the original work is properly cited.

\begin{abstract}
There is a lack of information about the methods used for bovine platelet-rich plasma (PRP)/platelet-rich gel (PRG) procurement, including information on platelet (PLT), white blood cell (WBC) in PRP, and growth factor release from PRG supernatants. The aims of this study were to compare and to correlate the PLT, WBC, transforming growth factor beta-1 (TGF- $\beta_{1}$ ), and plateletderived growth factor BB (PDGF-BB) concentrations in bovine whole blood, plasma, and four PRP layers and their respective PRG supernatants: A and B (obtained by a single centrifugation tube method at $720 \mathrm{~g} / 5 \mathrm{~min}$ ) and C and D (obtained by a double centrifugation tube method, by using two centrifugation episodes at $720 \mathrm{~g} / 5 \mathrm{~min}$ ). PLT and WBC counts were significantly higher in PRP-C, followed by whole blood, PRP-A, PRP-B, and PRP-D. TGF- $\beta_{1}$ concentrations were significantly higher in PRG-B supernatants and its correspondent PRP-B lysate when compared to the other PRG supernatants and plasma. Supernatants from PRG-A, PRG-B, and PRG-D had equivalent TGF- $\beta_{1}$ concentrations. PDGF-BB concentrations were not statistically different between the hemoderivatives. Significant Pearson correlations were noted between PLT counts and WBC counts (0.8) and between PLT counts and PLT distribution width (0.6). Further studies should be performed to assess the potential clinical applications of these PRPs.
\end{abstract}

\section{Introduction}

Platelet-rich plasma (PRP), a plasma preparation with variable concentrations of platelets (PLTs) and white blood cells (WBCs), is a biomaterial rich in growth factors (GFs), such as transforming growth factor beta-1 (TGF- $\beta_{1}$ ) and plateletderived growth factor type BB (PDGF-BB) [1]. Currently, PRP (liquid) compounds are classified into two groups according to the presence or absence of WBCs, that is, leukocyteconcentrated PRP (Lc-PRP) and leukocyte-reduced PRP (LrPRP), also known as pure-PRP (P-PRP) [2]. Once these PRPs are mixed with a platelet activating substance (i.e., calcium salt or thrombin) or come into contact with tissue collagen, they change into a gel form, that is, platelet-rich gel (PRG), which gradually releases GF. Notably, the cell composition of the PRP will determine the GF and cytokine profile released from activated PLTs and WBCs [3].

PRP types, either Lc-PRP or Lr-PRP, are currently used (among other uses) for the treatment of several musculoskeletal injuries and as a surgical coadjuvant in both human [4] and veterinary [5] medicine. In general, the rationale behind the use of this biomaterial lies in the fact that the GF and cytokines contained within are gradually released and diminish inflammation, promote neovascularization, increase extracellular matrix protein deposition, and induce cell migration and proliferation $[4,6]$. In other words, these substances could enhance tissue regeneration [7].

PRP has been mostly used in horses $[6,8]$ and dogs $[9,10]$ for the treatment of tenodesmic lesions and joint diseases. Furthermore, there are studies about the use of several PRPrelated hemoderivatives for the treatment of mastitis [11], reproductive problems [12], and sole ulcers [13] in cows with encouraging results. The basic biology of PRP (mostly cellular and molecular studies) has been more extensively studied in horses [14-18] and to a lesser degree in dogs [19-21]. However, to the best of our knowledge, there are only two studies describing a double centrifugation tube method for concentrating bovine platelets, in which a complete cellular profile of the procured PRP was described [22] and the 
influence of two anticoagulants on cell concentration was also analyzed [23]. However, there is scarce information about platelet-associated growth factors such as TGF- $\beta_{1}$ and PDGF$\mathrm{BB}$ in PRPs derived from cow blood.

In the present study, we evaluated a previously described double centrifugation tube method to obtain bovine PRP [22], which consisted in spin citrated whole blood at $720 \mathrm{~g}$ over $5 \mathrm{~min}$. After this, the plasma obtained was divided into PRP-A (the first 50\% plasma fraction nearest to the buffy coat) and PRP-B (the 50\% plasma fraction over PRP-A). PRPA was deposited in plain tubes, which were centrifugated at $720 \mathrm{~g}$ over $5 \mathrm{~min}$. After this, the first $25 \%$ plasma (bottom) fraction was considered as PRP-C and the remaining 75\% plasma (top) fraction was considered as PRP-D.

The novelty of this research stems from the fact that we analyzed, compared, and correlated cell numbers and TGF- $\beta_{1}$ and PDGF-BB concentrations in four different PRPs (platelet concentrates) which were produced over the entire process to obtain a final PRP fraction richer in PLTs than the other three evaluated PRP fractions or by-products. This basic study was performed to characterize from the cellular and molecular point of view four bovine PRP layers (A, B, C, and D) and to potentially recommend the use of a specific PRP for particular clinical problems in this species.

\section{Materials and Methods}

This study was approved by the committee for ethical experimentation with animals at Universidad de Caldas.

2.1. Animals. Nineteen clinically healthy lactating HolsteinFriesian cows with a median age of 5.5 years (range: $2.5-12$ years) were used. The animals were grazing in the highlands (2300 meters above sea level) of the central Andean region of Colombia. All the animals were fed and managed under similar technical conditions. The owner of the cows was informed on the nature of the study and signed an authorization accordingly.

2.2. Blood Collection. Blood was collected from each cow through a $2^{\prime \prime} 14 \mathrm{G}$ i.v. catheter (NIPRO14GX2", Nipro, São Paulo, Brazil), which was fixed with sutures in one of the jugular veins. The catheters were closed with a latexfree plug (IN-Stopper, B. Braun, Melsungen, Germany) and washed with $5 \mathrm{~mL}$ heparinized serum. Blood was obtained by attaching a $21 \mathrm{G}$ (Blood Collection Set, BD Vacutainer, New Jersey) butterfly catheter to the stopper [22]. The blood from each cow was deposited in $4.5 \mathrm{~mL}$ tubes containing $3.2 \% \mathrm{w} / \mathrm{v}$ sodium citrate (BD Vacutainer, New Jersey, USA). The first tube collected was discarded to avoid dilution or the biochemical effect of heparinized serum. The tubes containing blood were maintained in an icebox and transported to the laboratory where they were processed within 1 hour of being received.

2.3. Preparation of PRPs by Single Centrifugation Tube Method. Blood tubes with sodium citrate were centrifuged (Hettich Rotofix 32, Tuttlingen, Germany) at $720 \mathrm{~g}$ for 5

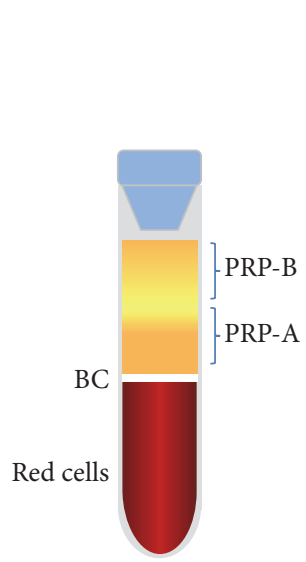

(a)

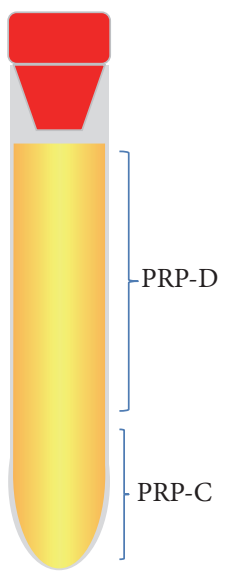

(b)
FIGURE 1: Schematic representation of the four platelet-rich plasma (PRP) fractions obtained with the single (A and B) and double (C and D) centrifugation tube methods. (a) Sodium citrate tube containing whole blood. After centrifugation, whole blood was separated in several components: red cells (PCV), buffy coat (BC), and the PRP-A (50\%) and PRP-B (50\%) fractions. (b) A $10 \mathrm{~mL}$ sterile plastic tube containing PRP-A. After centrifugation, this hemoderivative was divided into PRP-C (25\%) and PRP-D (75\%) fractions.

minutes [22]. The centrifugation process allowed for separating the blood into three phases: pack cell volume (PCV), the WBC layer, that is, the "buffy coat" (BC), and plasma. Plasma was divided into two PRP fractions: PRP-A and PRPB. Platelet-rich plasma fraction A corresponded to the $50 \%$ of plasma closest to the BC, whereas PRP-B was considered as the $50 \%$ of plasma immediately above PRP-A (Figure 1(a)). PRP-A was collected with the needle of a $2^{\prime \prime} 14 \mathrm{G}$ intravenous catheter (NIPRO14GX2", Nipro, São Paulo, Brazil), coupled to a $20 \mathrm{~mL}$ plastic syringe. A total of $10 \mathrm{~mL}$ of PRP-A and PRP$\mathrm{B}$ was obtained from each cow for this part of the study.

2.4. Preparation of PRPs by Double Centrifugation Tube Method. $20 \mathrm{~mL}$ of PRP-A (obtained by single centrifugation protocol) from each cow was deposited into two sterile plastic tubes without an additive (Vacuette, Greiner BioOne, Kremsmünster, Austria), which were centrifuged at $720 \mathrm{~g}$ for 5 minutes [22]. PRP-C was obtained after removing $7.5 \mathrm{~mL}$ of the upper plasma fraction (PRP-D) from each tube (Figure 1(b)).

2.5. Design of the Study. Several aliquots from PRP-A, PRP$\mathrm{B}$, PRP-C, and PRP-D were obtained for hematological and GF measurement. Samples $(1 \mathrm{~mL})$ from all PRPs and whole blood were employed for hematological impedance analysis (Celltac- $\alpha$ MEK 6450, Nihon Kohden, Japan), which included PLT and WBC counts and the determination of PLT-related activation parameters: mean PLT volume (MPV) and PLT distribution width (PDW). A $3 \mathrm{~mL}$ aliquot of each PRP was activated with $300 \mu \mathrm{L}$ of a calcium gluconate solution (9.3 mg/mL) (Ropshon Lab, Bogotá D.C., Colombia) 
in a 10:1 ratio. This procedure was done to induce PRG formation and subsequent release of GF. PRG samples were maintained with calcium gluconate under incubation at $37^{\circ} \mathrm{C}$ over $3 \mathrm{~h}$. After this, the plasma supernatant from PRGs (clots) was put into Eppendorf tubes and stored at $-80^{\circ} \mathrm{C}$ for later determination of GFs.

An additional $2 \mathrm{~mL}$ sample from all PRPs was incubated at $37^{\circ} \mathrm{C}$ for 15 minutes with $200 \mu \mathrm{L}$ of a solution containing $0.5 \%$ of a nonionic detergent (Triton ${ }^{\circledR}$ X-100, Sigma-Aldrich Co. LLC, MO, USA) for obtaining PRP lysates, which were used as a positive control of GF release. PRP lysates were processed in a similar fashion to PRG supernatants. Finally, $2 \mathrm{~mL}$ of plasma (free of cells) from each cow was also obtained by centrifugation of one blood tube at $3500 \mathrm{~g}$ for $5 \mathrm{~min}$. This substance was considered as the negative control for GF enrichment. Plasma samples were stored in the same fashion as PRG supernatants and PRP lysates.

2.6. Determination of TGF- $\beta_{1}$ and PDGF-BB Concentrations by ELISA. The PDGF-BB and TGF- $\beta_{1}$ concentrations in PRG supernatants, PRP lysates, and plasma were determined in duplicate by sandwich ELISA developed with commercial antibodies for human TGF- $\beta_{1}$ (Human TGF- $\beta 1$, DY240E, R\&D Systems, Inc., MN, USA) and PDGF-BB (Human PDGF-BB, DY220, R\&D Systems, Inc., MN, USA), because these mammalian GFs are highly homologous between both species (90\% identity or more) and similar ELISA antibodies have been used to measure these polypeptides in cow samples [24-27]. Both ELISAs were performed according to the manufacturer's instructions. Readings were performed at $450 \mathrm{~nm}$. Results are presented as pg of GF per $\mathrm{mL}$ of hemoderivative evaluated.

2.7. Statistical Analysis. Data were analyzed using statistical software (SPSS 18.0, IBM, Chicago, IL, USA). The ShapiroWilk test (SW) was used to evaluate the normality of the data. PLT and WBC counts and MPV and PDW parameters in whole blood and PRPs showed a normal distribution (SW, $p>0.05$ ) and were compared by one-way ANOVA, followed when necessary by a Tukey test. PDGF-BB concentrations in all hemoderivatives exhibited a parametric distribution (SW, $p>0.05)$. TGF- $\beta_{1}$ concentrations in some hemoderivatives presented a nonparametric distribution (SW, $p<0.05$ ). These data were analyzed after a $\log (Y)$ transformation. GF concentrations were compared by a generalized linear model (GLM) because there were some missing data for the concentration of both GFs. Notably, this procedure groups the dependent variables by their pattern of missing values across observations so that sums and cross-products can be collected in the most efficient manner. A Tukey test was used as a post hoc test when necessary. Furthermore, all variables were analyzed for general and specific correlations using a Pearson $\left(r_{s}\right)$ test. A $p$ value $\leq 0.05$ was considered significant for all the tests.

\section{Results}

3.1. Hematological Findings in Whole Blood and PRPs. PLT counts were significantly $(p=0.001)$ different between whole

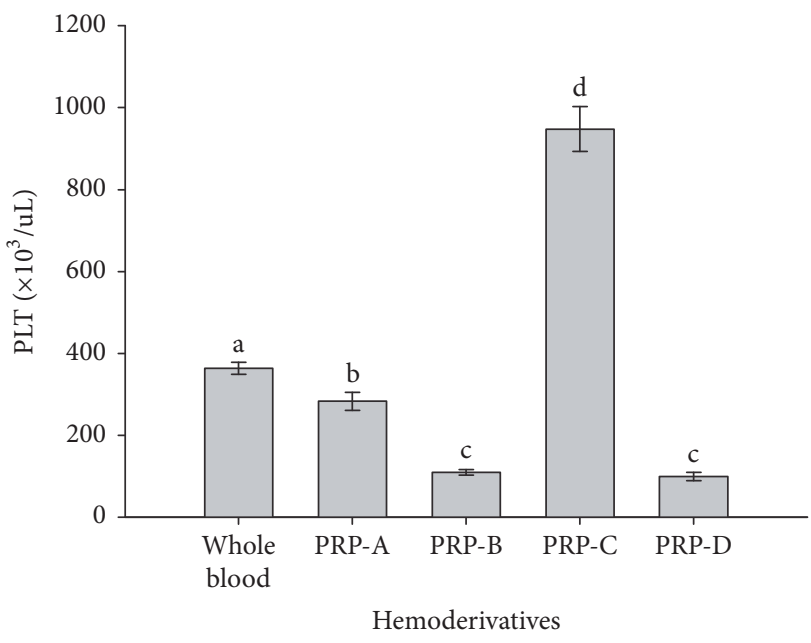

FIGURE 2: Mean ( \pm standard deviations [SD]) of platelet (PLT) concentration $\left(\times 10^{3} / \mu \mathrm{L}\right)$ in whole blood and four PRP fractions (A, B, C, and D). a-d: different lowercase letters denote significant differences by the Tukey test $(p=0.001)$.

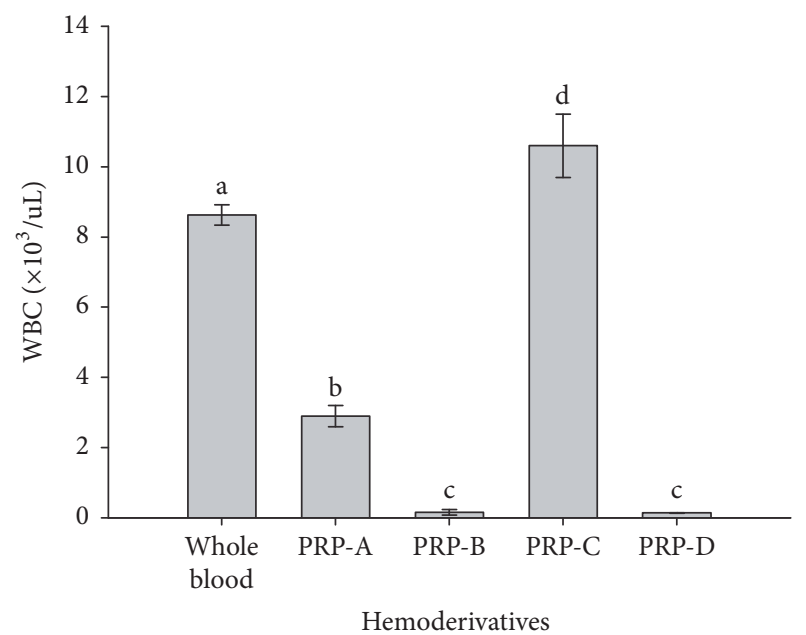

Figure 3: Mean $( \pm \mathrm{SD})$ of white blood cell (WBC) concentration $\left(\times 10^{3} / \mu \mathrm{L}\right)$ in whole blood and four bovine PRP fractions $(\mathrm{A}, \mathrm{B}, \mathrm{C}$, and $D)$. a-d: different lowercase letters denote significant differences by the Tukey test $(p=0.001)$.

blood and all PRPs. PRP-C showed a significantly ( $p=0.001)$ higher PLT concentration when compared to whole blood and the other PRPs. PRP-A showed a significantly $(p=0.001)$ lower PLT concentration when compared to whole blood and PRP-C and a higher concentration in comparison to PRP$\mathrm{B}$ and PRP-D, in which PLT counts were similar (Figure 2). On the other hand, WBCs showed a similar concentration pattern in whole blood and PRPs, which was very similar to those results' pattern observed for PLTs counts for the same hemoderivatives (Figure 3).

MPV values were not different between whole blood, PRP-A, and PRP-C. However, the values for the same parameter were significantly ( $p=0.001$ ) lower for PRP-B and PRP-D when compared to the other hemoderivatives and 


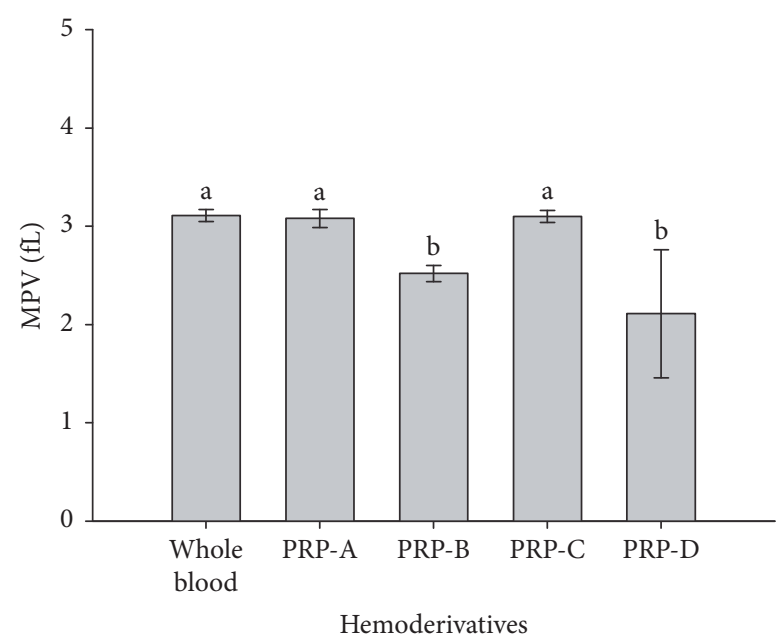

FIGURE 4: Mean $( \pm \mathrm{SD})$ of mean platelet volume (MPV) (fL) in whole blood and four bovine PRPs (A, B, C, and D). a-b: different lowercase letters denote significant differences by the Tukey test $(p=0.001)$.

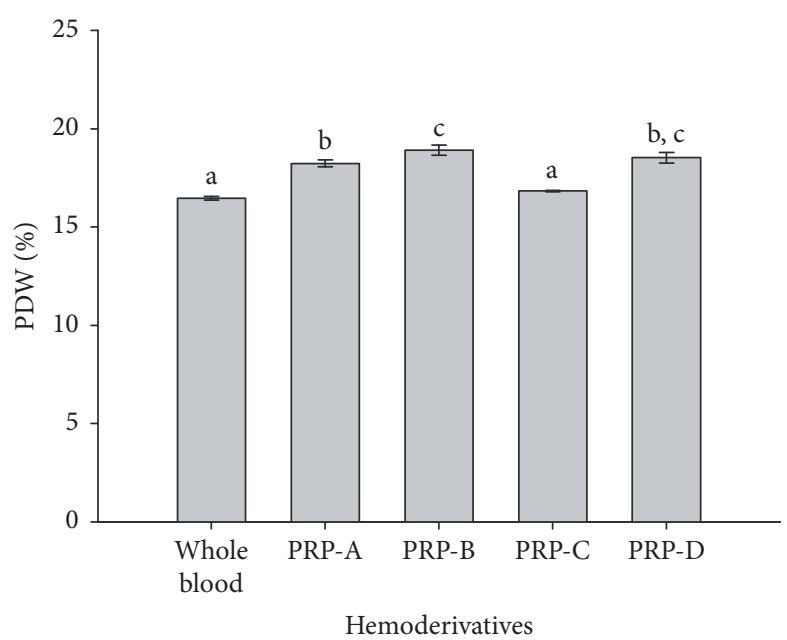

FIGURE 5: Mean $( \pm S D)$ of platelet distribution width (PDW) $(\%)$ in whole blood and four bovine PRPs (A, B, C, and D). a-c: different lowercase letters denote significant differences by the Tukey test $(p=$ $0.001)$.

whole blood (Figure 4). On the other hand, PDW values were significantly $(p=0.001)$ higher in PRP-B and PRP-D in comparison to whole blood and PRP-C, whereas PDW values were not different between PRP-B and PRP-D and between whole blood and PRP-C (Figure 5).

3.2. TGF- $\beta_{1}$ and PDGF-BB Concentrations in Plasma, PRG Supernatants, and PRP Lysates. TGF- $\beta_{1}$ concentrations were significantly $(p=0.001)$ lower in plasma when compared with the other hemoderivatives. On the other hand, the PRP$C$ lysate showed the highest TGF- $\beta_{1}$ concentration when compared to the rest of the hemoderivatives. Supernatants from PRG-A, PRG-B, and PRG-D were not different for this

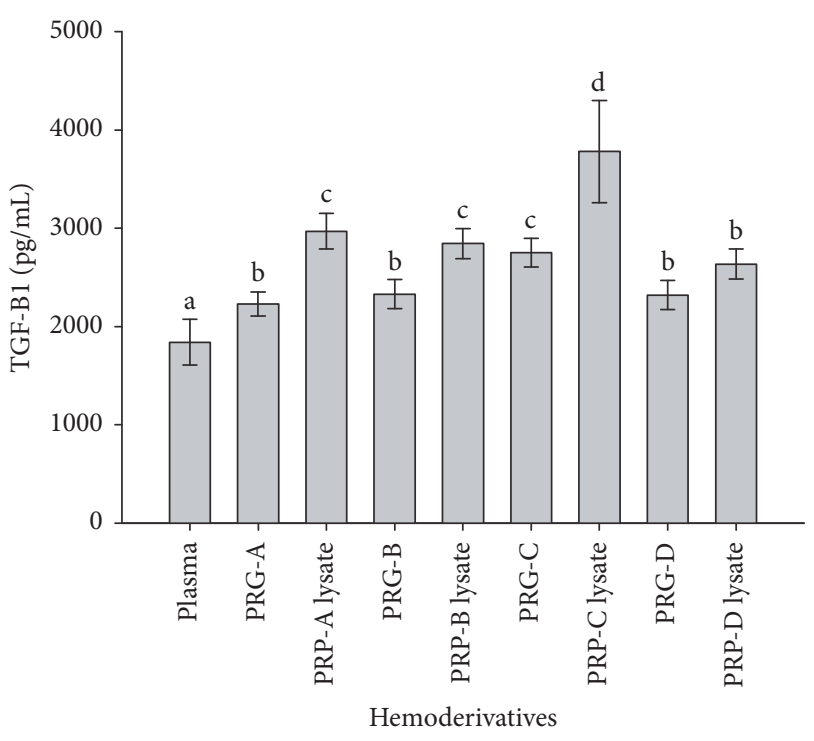

FIgURE 6: Mean $( \pm \mathrm{SD})$ of TGF- $\beta_{1}$ concentration $(\mathrm{pg} / \mathrm{mL})$ in plasma and four bovine platelet-rich gel (PRG) supernatants (A, B, C, and D) and their respective PRP lysates (A, B, C, and D). a-d: different lowercase letters denote significant differences by the Tukey test $(p<$ $0.05)$.

GF concentration. When each PRG supernatant was compared for its TGF- $\beta_{1}$ concentration in relation to the respective PRP lysate, we found significant differences between the PRG-A supernatant and PRP-A lysate ( $p=0.003)$, the PRGB supernatant and PRP-B lysate ( $p=0.001)$, and the PRG$C$ supernatant and PRP-C lysate $(p=0.0001)$. Notably, the TGF- $\beta_{1}$ concentration in the PRG-C supernatants was significantly ( $p=0.0001)$ higher in comparison with the other PRG supernatants (Figure 6). Missing values for this GF were observed for $2 \%$ of the generated data.

PDGF-BB concentrations remained at a concentration oscillating between 600 and $850 \mathrm{pg} / \mathrm{mL}$ in all hemoderivatives evaluated. No significant differences were noted for the concentration of this GF between the hemoderivatives (Figure 7). Missing values for this GF were observed for 3\% of the generated data.

3.3. Correlations. Significant correlations $\left(r_{s}\right)$ were noted between PLT counts and WBC counts $\left(r_{s}=0.80, p=\right.$ $0.0001)$, as well as between PLT counts and PDW $\left(r_{s}=-0.60\right.$, $p=0.001)$. Several significant weak correlations were noted between WBC counts and PDGF-BB concentrations $\left(r_{s}=\right.$ $-0.50, p=0.001)$, between PLT counts and PDGF-BB concentrations $\left(r_{s}=-0.40, p=0.001\right)$, and between PDGF-BB and TGF- $\beta_{1}$ concentrations $\left(r_{s}=0.50, p=0.001\right)$.

\section{Discussion}

The study described here presents novel and complimentary $[22,23]$ information on the double centrifugation tube technique for producing bovine PRP. As mentioned above, to the best of our knowledge, there are two additional published techniques [11-13] for concentrating platelets in 


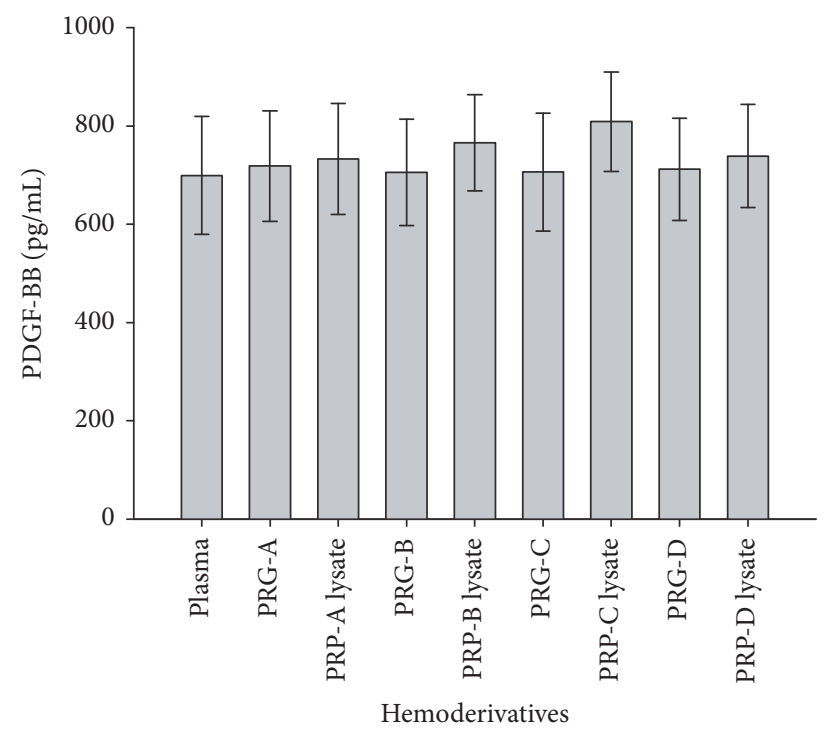

Figure 7: Mean $( \pm \mathrm{SD})$ of PDGF-BB concentration $(\mathrm{pg} / \mathrm{mL})$ in plasma and four bovine PRG supernatants (A, B, C, and D) and their respective PRP lysates (A, B, C, and D).

cattle. However, these studies did not provide information about WBC and GF concentrations. This information is of paramount importance to establish PRP protocols and, consequently, to understand their potential biological effects on cells and tissues [2, 28-30].

Currently, PRP classification is an important point of discussion for clinicians and researchers, because the cellular composition of a particular PRP will determine the content of GF and cytokines and consequently its biological effect $[2,28,30]$. Notably, 16 years ago, when seminal research work on PRP was published, there was evident interest in considering PRP as a $5 \mathrm{~mL}$ solution containing at least $1000 \times$ $10^{3} \mathrm{PLTs} / \mu \mathrm{L}$ [31]. At that time, it was thought that higher PLT concentrations in PRP would produce better clinical results than a substance with a lower PLT concentration. However, this paradigm has changed over time and, currently, it is widely accepted that PRP with a low PLT concentration $(\geq 1.2$ times greater than the PLT concentration in whole blood) can also produce good clinical results when compared to PRPs very rich in PLTs [32].

The results from the present study, particularly regarding the WBC concentration in PRPs, allowed us to classify the hemoderivatives into three types of liquid PRP compounds: (1) Lc-PRP (PRP-C), (2) Lr-PRP (PRP-B and PRP-D) [2], and (3) transitional PRP (T-PRP) (PRP-A). This last type of PRP is proposed as a PRP product characterized by a low count of WBCs (0.3-0.5 times the WBC concentration in whole blood) and a PLT concentration similar or slightly lower or higher in relation to the PLT concentration in whole blood [33]. At this point, it remains necessary to evaluate these bovine PRPs in in vitro systems and under clinical conditions affecting cattle. In general, these substances could be used as a treatment for orthopedic lesions, solar ulcers, mastitis, and traumatic wounds or as a surgical coadjuvant in this species.
PRP-C produced with the method evaluated in the present study presented a slightly lower concentration of PLTs $\left(947 \times 10^{3} \mathrm{PLTs} / \mu \mathrm{L}\right)$ when compared with the method described by Lange-Consiglio et al. [11, 12] who achieved $1000 \times 10^{3} \mathrm{PLTs} / \mu \mathrm{L}$. Furthermore, this same hemoderivative presented a lower concentration of PLTs when compared with the technique developed by Tsuzuki et al. [13] who achieved $1528 \times 10^{3} \mathrm{PLTs} / \mu \mathrm{L}$. On the other hand, the other PRP layers $(\mathrm{A}, \mathrm{B}$, and $\mathrm{D})$ evaluated in the present study presented lower PLT concentrations in comparison with those obtained in the aforementioned studies [11-13] and even in comparison to the PLT counts in whole blood of the same cows. Unfortunately, the WBC concentration was not reported for the techniques used for producing PRPs in cattle [11, 12, 29], which prevents us from comparing our findings with those reports.

MPV and PDW are frequently used parameters for indicating PLT activation in humans [34, 35], horses [17, 36], dogs $[37,38]$, and cattle [39], among others. These parameters are useful to determine PRP quality. However, the values for these parameters could change as a function of the anticoagulant used for blood collection and the technology of the device used for measurement $[15,38,40,41]$. Notably, MPV (fL) values in whole blood $(3.11 \pm 0.06)$ from the cows used in this study were lower than the same parameter reported in healthy cows in which whole blood $(6.30 \pm 0.17)$ was obtained with EDTA [39]. However, PDW (\%) values in whole blood $(16.47 \pm 0.10)$ from our cows were higher than the same value reported for whole blood $(10.70 \pm 0.30)$ of healthy cows using EDTA [39].

MPV values for all PRP layers evaluated in this study were similar between whole blood and PRP-A and PRP-C and significantly lower in PRP-B and PRP-D. This situation has also been observed in PRPs from horses using a similar technique [3]. One explanation for the changing MPV values, especially those related to PRP-B and PRP-D, is that these hemoderivatives presented a lower concentration of WBCs and erythrocytes. It is well known that PLTs become activated (reversibly) when they are centrifuged in the presence of these cells [42].

On the other hand, PDW (\%) values were significantly lower in whole blood and PRP-C in comparison with the other hemoderivatives. We also found a negative correlation $(-0.6)$ between this parameter and PLT counts in the PRPs. Possibly, PDW could be a more sensible indicator of PLT activation (PRP quality) than MPV in cattle, because an increase in this parameter was related to a lower concentration of PLTs in PRPs from the cows used in this study. It is important to consider that in humans PDW is a more specific marker for platelet activation than MPV, because it does not increase with only platelet swelling [41].

The inclusion of a negative control (plasma) and a positive control (PRP lysate) for establishing GF enrichment allowed us to observe that the supernatants from PRG-C and their correspondent PRP lysate showed the highest TGF$\beta_{1}$ concentrations. Notably, $73 \%$ of TGF- $\beta_{1}$ stored in PLTs and WBCs from PRP-C was massively released $3 \mathrm{~h}$ after activation. It seems that TGF- $\beta_{1}$ release from cattle PRP after its activation is more massive and rapid than in horses [17]. Notably, we found that TGF- $\beta_{1}$ concentrations were 
significantly higher in all hemoderivatives when compared to plasma. This finding suggests that all PRP layers evaluated contain important TGF- $\beta_{1}$ concentrations, indicating that these biomaterials have potential clinical application in this species.

PDGF-BB concentrations were similar between all hemoderivatives evaluated in the present study. This was an unexpected biological phenomenon, because this GF is regularly present in lower concentrations in plasma and in higher concentrations in PRG supernatants and their correspondent PRP lysates in mammals, like horses $[15,17,43]$, cats [44], and rabbits [33]. Two technical or biological explanations could explain this finding: (1) human PDGF-BB specific antibodies were used for capturing bovine PDGF-BB. As mentioned above, this GF is very similar between human and bovine [25]; however, similar PDGF-BB ELISA antibodies (from the same manufacturer) have been used for the same objective in other studies in which bovine PDGF-BB concentrations were measured [26, 27]. (2) It is possible that bovine PDGF-BB presented a high plasma concentration and a nonvariable concentration in PRP as a function of the PLT concentration. Notably, this biological phenomenon has also been observed in humans $[45,46]$ and horses $[43,47]$ for an important anabolic GF related to PRP, insulin growth factor type I. However, further research evaluating the concentrations of several bovine PDGF isoforms in PRP using species-specific ELISA antibodies should be performed.

\section{Conclusions}

This study demonstrates that several bovine PRPs can be obtained using single or double centrifugation tube methods. According to the results on cell counts, the PRP types could be classified as Lc-PRP (PRP-C), Lr-PRP (PRP-B and PRPD), or transitional PRP (T-PRP) (PRP-A). PRP-C presented the highest concentration of PLTs, WBCs, and TGF- $\beta_{1}$, followed by PRP-A, PRP-B, and PRP-D. However, PDGF-BB concentrations were similar between all hemoderivatives.

\section{Disclosure}

This manuscript represents a part of an M.S. thesis submitted to the Master of Veterinary Sciences Program at Universidad de Caldas, which was performed by the first author and supervised by Jorge U. Carmona.

\section{Conflicts of Interest}

No potential conflicts of interest were reported by the authors.

\section{Authors' Contributions}

Jorge U. Carmona had full access to all of the data in the study and takes responsibility for the decision to submit this work for publication. Jorge U. Carmona and Claudia M. Gutiérrez designed the study and participated in data analysis and interpretation, manuscript drafting, and critical revision. Claudia M. Gutiérrez, Catalina López, and Carlos E. Giraldo performed laboratory work and contributed to data collection, data analysis and interpretation, and critical revision of the manuscript. All of the authors read and approved the final version of the manuscript to be published.

\section{Acknowledgments}

This project was supported by a grant for developing of research groups of the Vicerrectoría de Investigaciones y Postgrados of the Universidad de Caldas, Manizales, Colombia. The authors would like to thank Sebastián Gutiérrez and James Borbón for their assistance.

\section{References}

[1] P. I.-K. Wu, R. Diaz, and J. Borg-Stein, "Platelet-Rich Plasma," Physical Medicine and Rehabilitation Clinics of North America, vol. 27, no. 4, pp. 825-853, 2016.

[2] D. M. Dohan Ehrenfest, T. Bielecki, A. Mishra et al., "In Search of a consensus terminology in the field of platelet concentrates for surgical use: platelet-Rich Plasma (PRP), Platelet-Rich Fibrin (PRF), fibrin gel polymerization and leukocytes," Current Pharmaceutical Biotechnology, vol. 13, no. 7, pp. 1131-1137, 2012.

[3] S. Arora, V. Doda, U. Kotwal, and M. Dogra, "Quantification of platelets and platelet derived growth factors from plateletrich-plasma (PRP) prepared at different centrifugal force (g) and time," Transfusion and Apheresis Science, vol. 54, no. 1, pp. 103-110, 2016.

[4] M. R. De Pascale, L. Sommese, A. Casamassimi, and C. Napoli, "Platelet derivatives in regenerative medicine: an update," Transfusion Medicine Reviews, vol. 29, no. 1, pp. 52-61, 2015.

[5] L. Corti, "Nonpharmaceutical approaches to pain management," Topics in Companion Animal Medicine, vol. 29, no. 1, pp. 24-28, 2014.

[6] P. M. Brossi, J. J. Moreira, T. S. Machado, and R. Y. Baccarin, "Platelet-rich plasma in orthopedic therapy: a comparative systematic review of clinical and experimental data in equine and human musculoskeletal lesions," BMC Veterinary Research, vol. 11, no. 1, pp. 98-148, 2015.

[7] J. C. González, C. López, M. E. Álvarez, J. E. Pérez, and J. U. Carmona, "Autologous leukocyte-reduced platelet-rich plasma therapy for Achilles tendinopathy induced by collagenase in a rabbit model," Scientific Reports, vol. 6, article 19623, 2016.

[8] M. Scala, S. Lenarduzzi, F. Spagnolo et al., "Regenerative medicine for the treatment of Teno-desmic injuries of the equine. A series of 150 horses treated with platelet-derived growth factors," In Vivo, vol. 28, no. 6, pp. 1119-1124, 2014.

[9] R. F. Silva, J. U. Carmona, and C. M. F. Rezende, "Intra-articular injections of autologous platelet concentrates in dogs with surgical reparation of cranial cruciate ligament rupture," Veterinary and Comparative Orthopaedics and Traumatology, vol. 26, no. 4, pp. 285-290, 2013.

[10] J. M. Vilar, M. Morales, A. Santana et al., "Controlled, blinded force platform analysis of the effect of intraarticular injection of autologous adipose-derived mesenchymal stem cells associated to PRGF-Endoret in osteoarthritic dogs," BMC Veterinary Research, vol. 9, pp. 1746-6148, 2013.

[11] A. Lange-Consiglio, C. Spelta, R. Garlappi, M. Luini, and F. Cremonesi, "Intramammary administration of platelet concentrate 
as an unconventional therapy in bovine mastitis: first clinical application," Journal of Dairy Science, vol. 97, no. 10, pp. 62236230, 2014.

[12] A. Lange-Consiglio, N. Cazzaniga, R. Garlappi et al., "Platelet concentrate in bovine reproduction: effects on in vitro embryo production and after intrauterine administration in repeat breeder cows," Reproductive Biology and Endocrinology, vol. 13, no. 1, article no. 65, 2015.

[13] N. Tsuzuki, J.-P. Seo, K. Yamada, S. Haneda, Y. Tabata, and N. Sasaki, "Effect of compound of gelatin hydrogel microsphere incorporated with platelet-rich-plasma and alginate on sole defect in cattle," The Journal of Veterinary Medical Science, vol. 74, no. 8, pp. 1041-1044, 2012.

[14] G. Hauschild, F. Geburek, G. Gosheger et al., "Short term storage stability at room temperature of two different platelet-rich plasma preparations from equine donors and potential impact on growth factor concentrations," BMC Veterinary Research, vol. 13, no. 1, article no. 7, 2017.

[15] C. E. Giraldo, M. E. Álvarez, and J. U. Carmona, "Influence of calcium salts and bovine thrombin on growth factor release from equine platelet-rich gel supernatants," VCOT, vol. 30, no. 1, pp. 1-7, 2017.

[16] R. Rinnovati, N. Romagnoli, F. Gentilini, C. Lambertini, and A. Spadari, "The influence of environmental variables on platelet concentration in horse platelet-rich plasma," Acta Veterinaria Scandinavica, vol. 58, no. 1, article no. 45, 2016.

[17] C. E. Giraldo, M. E. Álvarez, and J. U. Carmona, "Effects of sodium citrate and acid citrate dextrose solutions on cell counts and growth factor release from equine pure-platelet rich plasma and pure-platelet rich gel," BMC Veterinary Research, vol. 11, no. 1, article 60, 2015.

[18] L. N. Hessel, G. Bosch, P. R. van Weeren, and J.-C. Ionita, "Equine autologous platelet concentrates: a comparative study between different available systems," Equine Veterinary Journal, vol. 47, no. 3, pp. 319-325, 2015.

[19] B. J. Carr, S. O. Canapp, D. R. Mason, C. Cox, and T. Hess, "Canine Platelet-Rich Plasma Systems: a Prospective Analysis," Frontiers in Veterinary Science, vol. 2, 2016.

[20] C. W. Frye, A. Enders, M. B. Brooks, A. M. Struble, and J. J. Wakshlag, "Assessment of canine autologous platelet-rich plasma produced with a commercial centrifugation and platelet recovery kit," Veterinary and Comparative Orthopaedics and Traumatology, vol. 29, no. 1, pp. 14-19, 2016.

[21] R. F. Silva, J. U. Carmona, and C. M. F. Rezende, "Comparison of the effect of calcium gluconate and batroxobin on the release of transforming growth factor beta 1 in canine platelet concentrates," BMC Veterinary Research, vol. 8, pp. 1746-6148, 2012.

[22] C. López, C. E. Giraldo, and J. U. Carmona, "Evaluation of a double centrifugation tube method for concentrating bovine platelets: cellular study," Archivos de Medicina Veterinaria, vol. 44, no. 2, pp. 109-115, 2012.

[23] J. U. Carmona, E. Ríos, J. M. Vilar, C. E. Giraldo, and C. López, "Effect of two anticoagulants on the cell count and platelet activation parameters from bovine platelet rich plasma," Archivos de Medicina Veterinaria, vol. 46, no. 3, pp. 375-380, 2014.

[24] E. Van Obberghen-Schilling, P. Kondaiah, R. L. Ludwig, M. B. Sporn, and C. C. Baker, "Complementary deoxyribonucleic acid cloning of bovine transforming growth factor- $\beta 1$," Molecular Endocrinology, vol. 1, no. 10, pp. 693-698, 1987.
[25] S. Xie and S. P. Walton, "Development of a dual-aptamer-based multiplex protein biosensor," Biosensors and Bioelectronics, vol. 25, no. 12, pp. 2663-2668, 2010.

[26] A. Dardik, A. Yamashita, F. Aziz, H. Asada, and B. E. Sumpio, "Shear stress-stimulated endothelial cells induce smooth muscle cell chemotaxis via platelet-derived growth factor-BB and interleukin-1 $\alpha$, Journal of Vascular Surgery, vol. 41, no. 2, pp. 321-331, 2005.

[27] A. Moshiri, A. Oryan, and A. Meimandi-Parizi, "Synthesis, development, characterization and effectiveness of bovine pure platelet gel-collagen-polydioxanone bioactive graft on tendon healing," Journal of Cellular and Molecular Medicine, vol. 19, no. 6, pp. 1308-1332, 2015.

[28] Y. Kobayashi, Y. Saita, H. Nishio et al., "Leukocyte concentration and composition in platelet-rich plasma (PRP) influences the growth factor and protease concentrations," Journal of Orthopaedic Science, vol. 21, no. 5, pp. 683-689, 2016.

[29] H. Masuki, T. Okudera, T. Watanebe et al., "Growth factor and pro-inflammatory cytokine contents in platelet-rich plasma (PRP), plasma rich in growth factors (PRGF), advanced platelet-rich fibrin (A-PRF), and concentrated growth factors (CGF)," International Journal of Implant Dentistry, vol. 2, no. 1, 2016.

[30] M. O. Schär, J. Diaz-Romero, S. Kohl, M. A. Zumstein, and D. Nesic, "Platelet-rich concentrates differentially release growth factors and induce cell migration in vitro," Clinical Orthopaedics and Related Research, vol. 473, no. 5, pp. 1635-1643, 2015.

[31] R. E. Marx, "Platelet-rich plasma (PRP): what is PRP and what is not PRP?" Implant Dentistry, vol. 10, no. 4, pp. 225-228, 2001.

[32] T. Kawase, "Platelet-rich plasma and its derivatives as promising bioactive materials for regenerative medicine: basic principles and concepts underlying recent advances," Odontology, vol. 103, no. 2, pp. 126-135, 2015.

[33] J. C. González, C. López, and J. U. Carmona, "Implications of anticoagulants and gender on cell counts and growth factor concentration in platelet-rich plasma and platelet-rich gel supernatants from rabbits," Veterinary and Comparative Orthopaedics and Traumatology, vol. 29, no. 2, pp. 115-124, 2016.

[34] Y. U. Budak, M. Polat, and K. Huysal, "The use of platelet indices, plateletcrit, mean platelet volume and platelet distribution width in emergency non-traumatic abdominal surgery: a systematic review," Biochemia Medica, vol. 26, no. 2, pp. 178-193, 2016.

[35] M. A. AlSheeha, R. S. Alaboudi, M. A. Alghasham, J. Iqbal, and I. Adam, "Platelet count and platelet indices in women with preeclampsia," Vascular Health and Risk Management, vol. 12, pp. 477-480, 2016.

[36] R. L. Fontenot, C. A. Sink, S. R. Werre, N. M. Weinstein, and L. A. Dahlgren, "Simple tube centrifugation for processing platelet-rich plasma in the horse," Canadian Veterinary Journal, vol. 53, no. 12, pp. 1266-1272, 2012.

[37] A. Moritz, B. K. Walcheck, and D. J. Weiss, "Evaluation of flow cytometric and automated methods for detection of activated platelets in dogs with inflammatory disease," American Journal of Veterinary Research, vol. 66, no. 2, pp. 325-329, 2005.

[38] T. Stokol and H. N. Erb, "A comparison of platelet parameters in EDTA- And citrate-anticoagulated blood in dogs," Veterinary Clinical Pathology, vol. 36, no. 2, pp. 148-154, 2007.

[39] M. R. Abd Ellah, "Studying the correlations among hematological and serum biochemical constituents in cattle theileriosis," Journal of Parasitic Diseases, vol. 39, no. 2, pp. 134-139, 2015. 
[40] D. Argüelles, J. U. Carmona, J. Pastor et al., "Evaluation of single and double centrifugation tube methods for concentrating equine platelets," Research in Veterinary Science, vol. 81, no. 2, pp. 237-245, 2006.

[41] E. Vagdatli, E. Gounari, E. Lazaridou, E. Katsibourlia, F. Tsikopoulou, and I. Labrianou, "Platelet distribution width: a simple, practical and specific marker of activation of coagulation," Hippokratia, vol. 14, no. 1, pp. 28-32, 2010.

[42] J. K. Kingston, W. M. Bayly, D. C. Sellon, K. M. Meyers, and K. J. Wardrop, "Effects of sodium citrate, low molecular weight heparin, and prostaglandin E1 on aggregation, fibrinogen binding, and enumeration of equine platelets," American Journal of Veterinary Research, vol. 62, no. 4, pp. 547-554, 2001.

[43] L. V. Schnabel, H. O. Mohammed, B. J. Miller et al., "Platelet Rich Plasma (PRP) enhances anabolic gene expression patterns in flexor digitorum superficialis tendons," Journal of Orthopaedic Research, vol. 25, no. 2, pp. 230-240, 2007.

[44] R. F. Silva, M. E. Álvarez, D. L. Ríos, C. López, J. U. Carmona, and C. M. F. Rezende, "Evaluation of the effect of calcium gluconate and bovine thrombin on the temporal release of transforming growth factor beta 1 and platelet-derived growth factor isoform BB from feline platelet concentrates," BMC Veterinary Research, vol. 8, article no. 212, pp. 1746-6148, 2012.

[45] P. A. M. Everts, C. Brown Mahoney, J. J. M. L. Hoffmann et al., "Platelet-rich plasma preparation using three devices: implications for platelet activation and platelet growth factor release," Growth Factors, vol. 24, no. 3, pp. 165-171, 2006.

[46] G. Schmidmaier, S. Herrmann, J. Green et al., "Quantitative assessment of growth factors in reaming aspirate, iliac crest, and platelet preparation," Bone, vol. 39, no. 5, pp. 1156-1163, 2006.

[47] W. W. Sutter, A. J. Kaneps, and A. L. Bertone, "Comparison of hematologic values and transforming growth factor- $\beta$ and insulin-like growth factor concentrations in platelet concentrates obtained by use of buffy coat and apheresis methods from equine blood," American Journal of Veterinary Research, vol. 65, no. 7, pp. 924-930, 2004. 

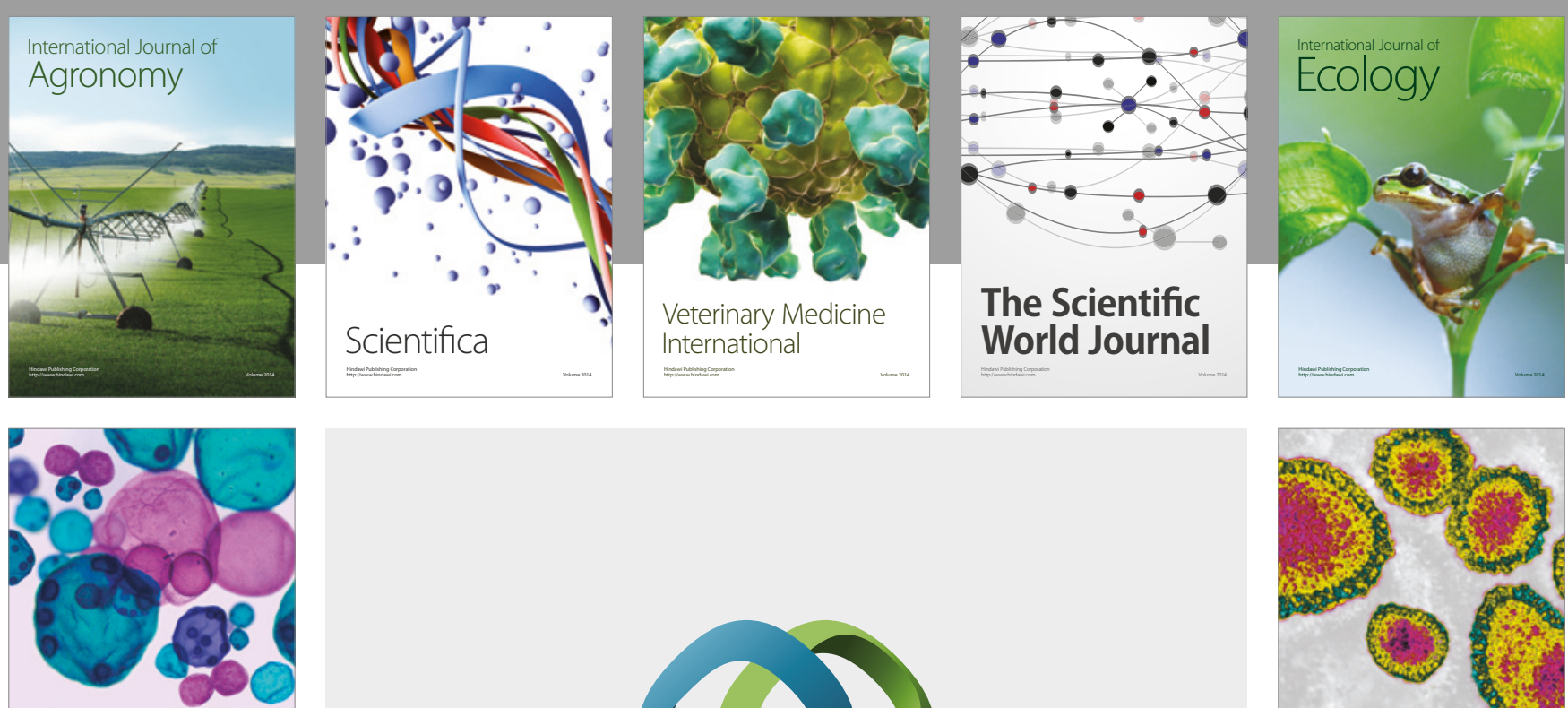

\section{International Journal of} Microbiology
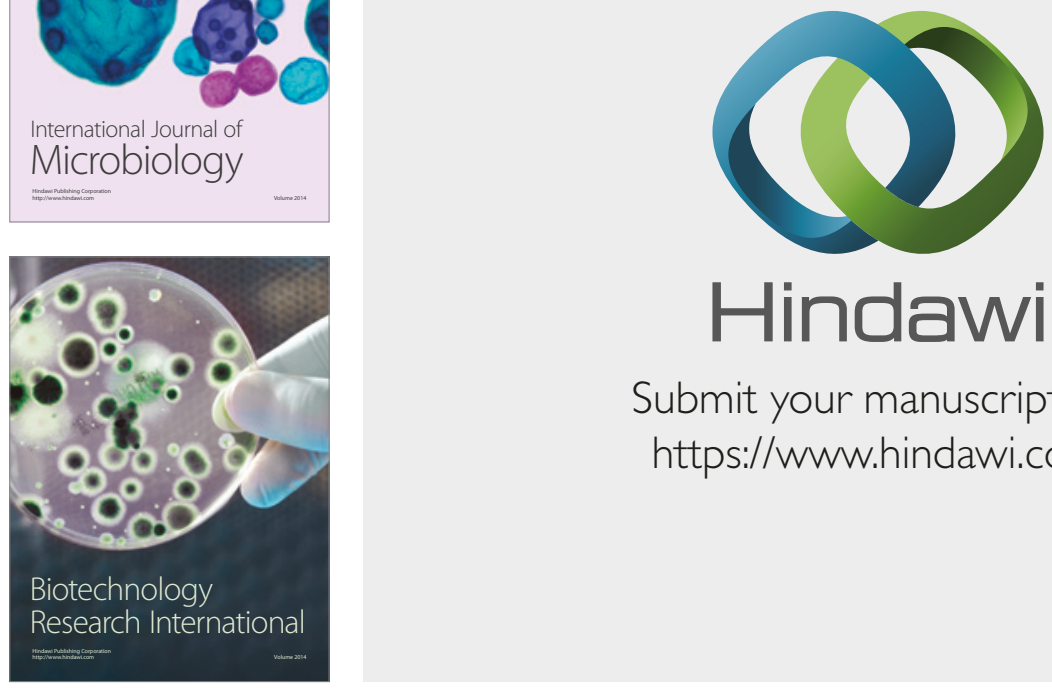

Submit your manuscripts at

https://www.hindawi.com
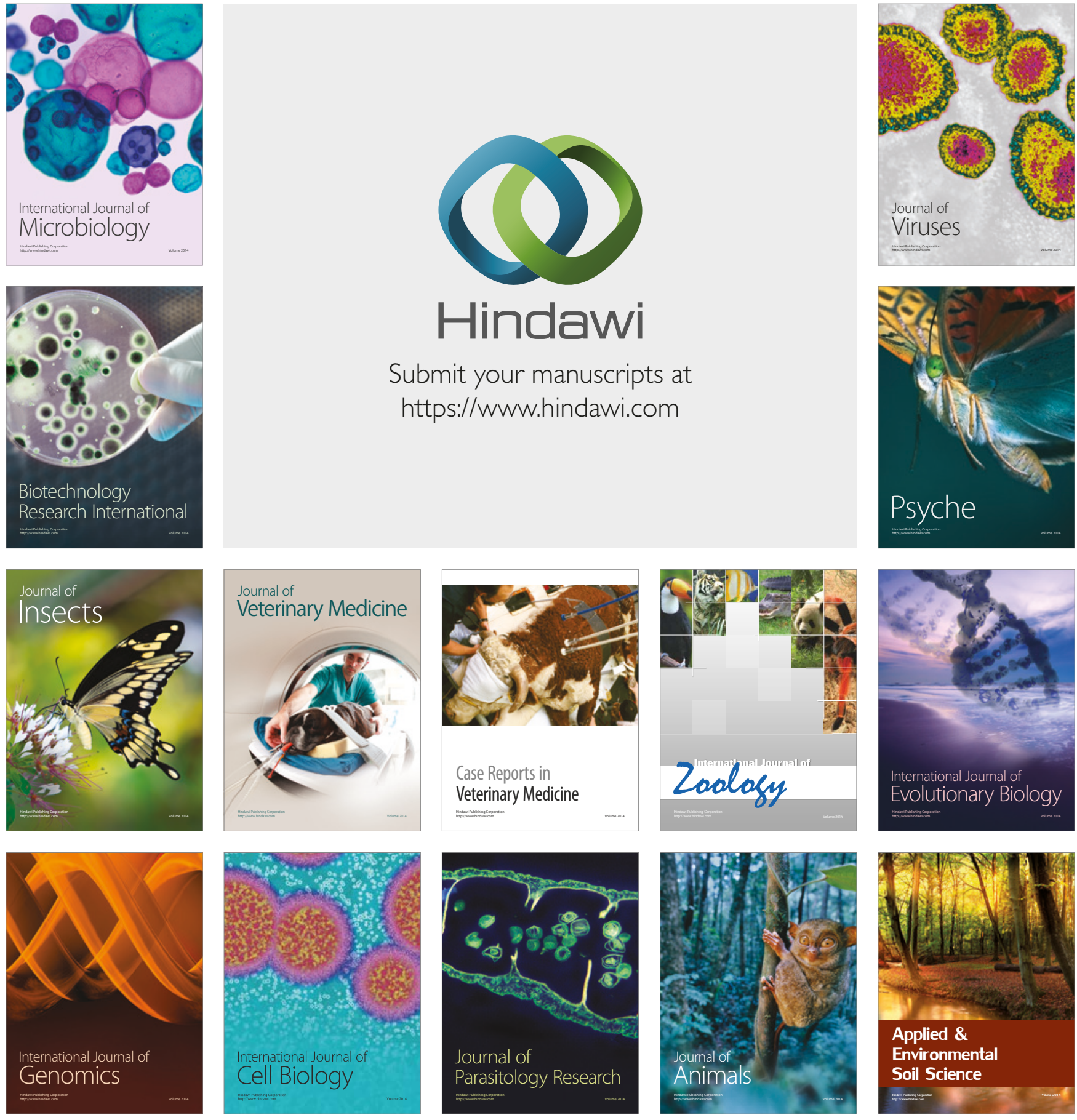